\title{
Distribution and correlation of psychiatric symptoms in early stages of Parkinson's disease
}

\author{
Nicoleta Tohanean, Catalina Crisan, Lacramioara Perju-Dumbrava \\ Neuroscience Department, "Iuliu Hatieganu" University of Medicine and Pharmacy, Cluj-Napoca Romania
}

\begin{abstract}
The premotor phase of Parkinson Disease (PD) precedes the classical motor phase, and the clinical picture is dominated by nonmotor symptoms. The psychiatric symptoms, an important category of nonmotor symptoms, need to be evaluated and treated.

The study objective was to assess the frequency of nonmotor symptoms in patients with PD in early stages of the disease and to establish correlations between these symptoms and the features of disease or clinical characteristics of patients.

The group of patients enrolled in the study included a number of 43 patients with early PD, enrolled during a period of one year in Neurology Clinic I, from Cluj-Napoca, Romania. For all patients enrolled in the study, the questionnaire NMS-PD QUEST was applied.

Psychiatric complaints play an important role as there were no patients who did not present at least one psychiatric symptom, and, in most cases, patients have described a complex of associated symptoms: roughly one third of the patients of both genders complain about 5 symptoms of each of this type, simultaneously. Within the psychiatric field, the most prevalent symptoms belonged to affective disorders- depression-81,39\%, followed by disorders of attention and concentration-79, 06\%. Regarding the correlations monitored in the study, the analysis of parameters shows that the only statistically significant correlation was with family history of PD. The field of sleep disorders was well represented by insomnia, present in $69.76 \%$ of cases, which was followed by excessive daytime sleepiness and sleep with nightmares in equal percentages of $46.51 \%$.

The most common psychiatric symptoms reported by the questionnaire even from the early stages were depression, attention and memory problems and those related to impaired sleep.

The results presented highlights the importance of questioning the patients about nonmotor complaints from the very beginning of the diagnostic process.
\end{abstract}

Keywords: nonmotor symptoms, depression, sleep disorders, Parkinson disease

\section{INTRODUCTION}

The recent years of clinical, pathogenetic and pathological studies have revolutionized the traditional concepts of PD as a motor entity, talking more about premotor phase who precedes the classical motor phase and when the clinical picture is dominated by nonmotor symptoms (1).

Also recent literature data describe the syndrome at risk of developing Parkinson diseasesyndrome PARS (2), which includes this prediagnostic disease phase, that is represented by clinical, genetic and imaging markers, attemptting to establish the diagnosis in order to initiate a neuroprotective therapy $(3,4)$.
Nonmotors symptoms of PD are varied, numerous and nonspecific, which are present even in the preclinical stages of disease and which could contribute to early diagnosis of the disease $(1,5)$. These premotors symptoms are represented by olfactory dysfunction, autonomic dysfunction (constipation, orthostatic hypotension), sleep disorders (RBD) and depression $(6,7)$.

The psychiatric symptoms represent a important category of premotors symptoms, important to be evaluated and treated.

Deppresion is common in $\mathrm{PD}$, with a incidence of $32 \%$ in PD early diagnosed. In $44 \%$ of cases it preceded motor symptoms until 20 years before, 
so is considered a premotor symptom (8). Patients with depression have a $\mathbf{2 , 2 - 3 , 2}$ higher risk to develop PD and is associated with important impairment of activities of daily living (9).

Anxiety is frequent in both early and advanced stages of disease and coexists with depression. Her prevalence is $62,9 \%$ in early stages of $\mathbf{P D}$ and is not correlated with duration of the disease, the severity of motor symptoms, the dose of L-Dopa or degree of disability (8).

RBD (REM behaviour disorder) is a parasomnia, defined as recurrent episodes of strong abnormal movements of the body, head or limbs occurring during REM sleep, often associated with dreams when the patient is defending against aggression $(10,11)$. Prevalence of RBD in PD is not known exactly but studies show that at least $\mathbf{3 0 \%}$ of patients with a clinical diagnosis of PD have clinical or polysomnographic features of RBD (12). RBD can precede motor symptoms a few years, so is a premotor symptom (13). RBD in patients with BP appears to be a risk factor for the development of cognitive impairment and dementia (14).

Excessive daytime sleepiness (EDS) is defined as "being sleepy most of the day" or as "episodes of sudden irresistible sleepiness during current daily activities". The frequency is varying between 15 and $71 \%$ of PD patients and is $\mathbf{1 , 5}$ up to 15 times more frequently in PD than healthy subjects (8). Dopaminergic agents drugs increase significantly dose-dependent) the risk of development of EDS (8). EDS correlates with severity of motor impairment and cognitive decline and is a risk factor for PD (increases the risk 3 times) $=$ premotor symptom $(8,15)$.

\section{MATERIAL AND METHOD}

The group of patients enrolled in the study included a number of $\mathbf{4 3}$ patients, who where either consecutive hospitalized or being reffered from ambulatory consultation during a period of one year (1.01.2010-31.12.2010) in Neurology Clinic I, from Cluj-Napoca, Romania (17).

These patients were either presenting a clinical onset of parkinsonian syndrome, or where diagnosed with early PD (duration of up to 3 years after diagnosis, PD stages of disease 1, 1.5 and 2 Hoehn \& Yahr - H\&Y).
For all patients enrolled in the study were applied the questionnaire NMS-PD QUEST (7) and were identified the nonmotors symptoms, setting their frequency and classification on areas of involvement. The questionnaire performed has pursued 7 psychiatric symptoms and 4 sleep disturbances.

\section{RESULTS (17)}

Psychiatric disorders are often found in patients with PD at all stages. Fig. 1 illustrate the distribution of the main psychiatric symptoms that occur in the early stages of the disease. It observed high prevalence of depression $(81.39 \%)$, followed by disorders of attention (79.06\%) and memory (74.41\%). Over about a half of patients complain of anxiety disorders $(60.46 \%)$ and loss of interest in usual activities-ahedonie (53.48\%) and a smaller number of patients presents illusions (23.25\%) and hallucinations (16.27\% ). In our sample, all psychiatric symptoms had a higher incidence in women.

The questionnaire conducted followed 7 psychiatric symptoms and the most commonly, patients described more symptoms associated. Only $15.38 \%$ of women and $5.88 \%$ of men had only a psychiatric symptom. Approximately one third of patients, respectively $30.77 \%$ of women and $35.29 \%$ of men accuse simulteneus 5 psychiatric symptoms. A small number of patients- under $10 \%$ accuse 5 or 6 psychiatric symptoms and the rest present only 2.3 or 4 concomitant symptoms in percentages ranging between 10 and $20 \%$. There were no patients who does not present psychiatric impairment.

At statistical analysis performed, independent samples t-test (with coefficient $p$ calculation ( $p$ is significant to $<0.05)$ ) was used to establish correlations between psychiatric symptoms with patient gender, origin, smoking or toxic exposure, family history, type of treatment. To analyze the correlations between psychiatric symptoms and disease duration of patients' age it has been used parametric Pearson correlation (r). For comparing symptoms according to the stage and type of disease it has been used analysis of unifactorial variance ANOVA.

The psychiatric disorders have not varied according to the gender of the patient $(t=.00 ; p=1)$, the environment $(\mathrm{t}=-.08 ; \mathrm{p}=0.94)$, smoking $(\mathrm{t}=.69$; 


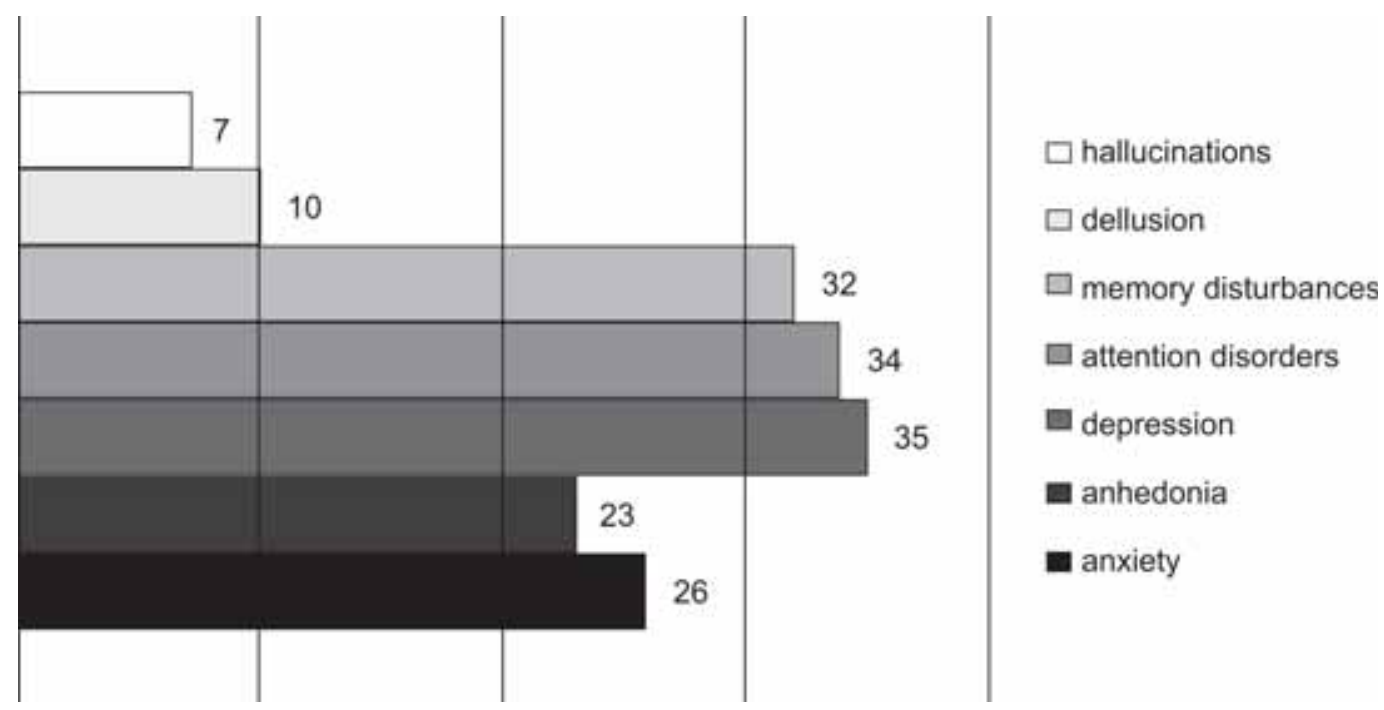

FIGURE 1. Frequency of psychiatric symptoms

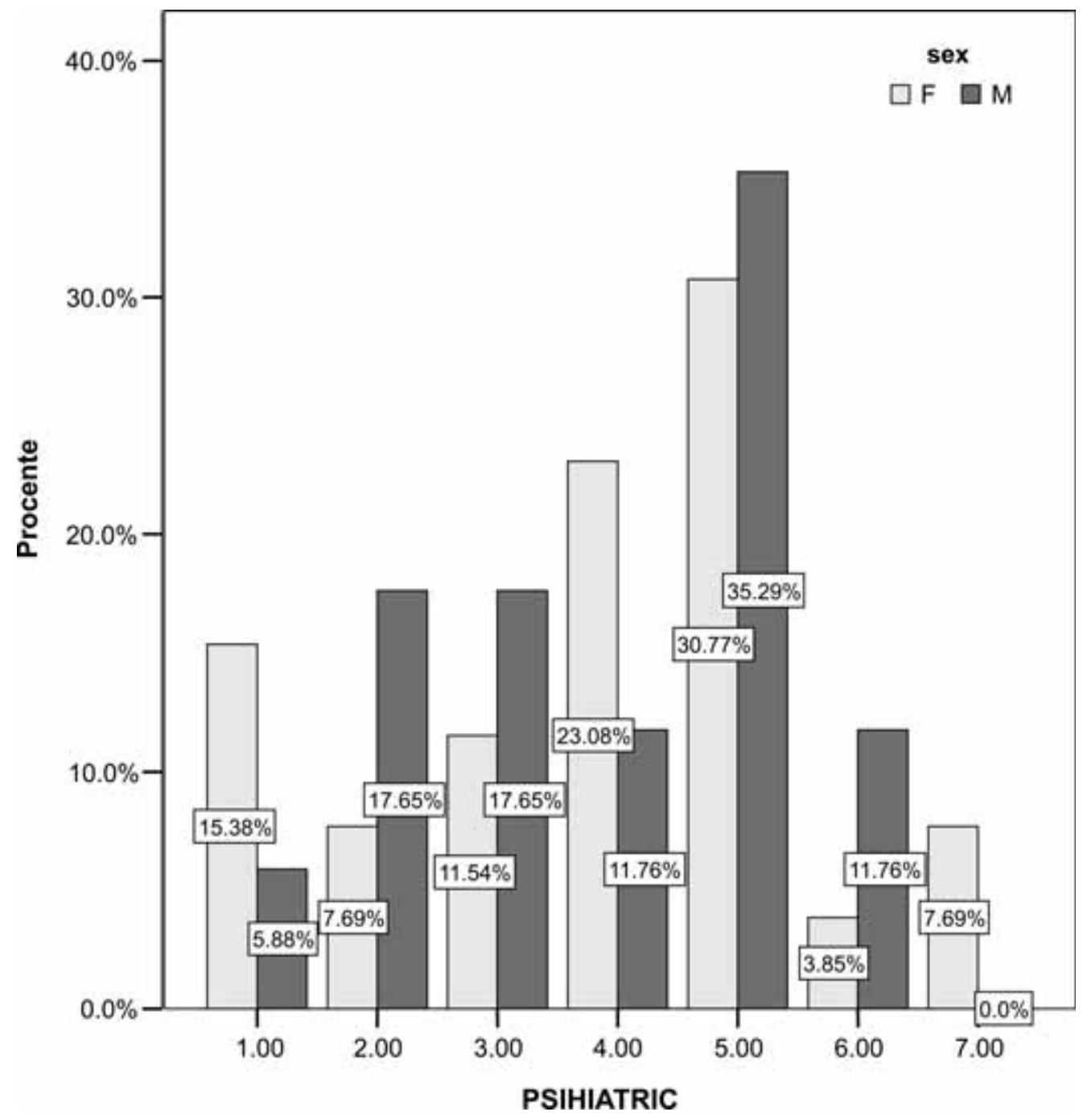

FIGURE 2. Distribution of psychiatric symptoms according to gender 


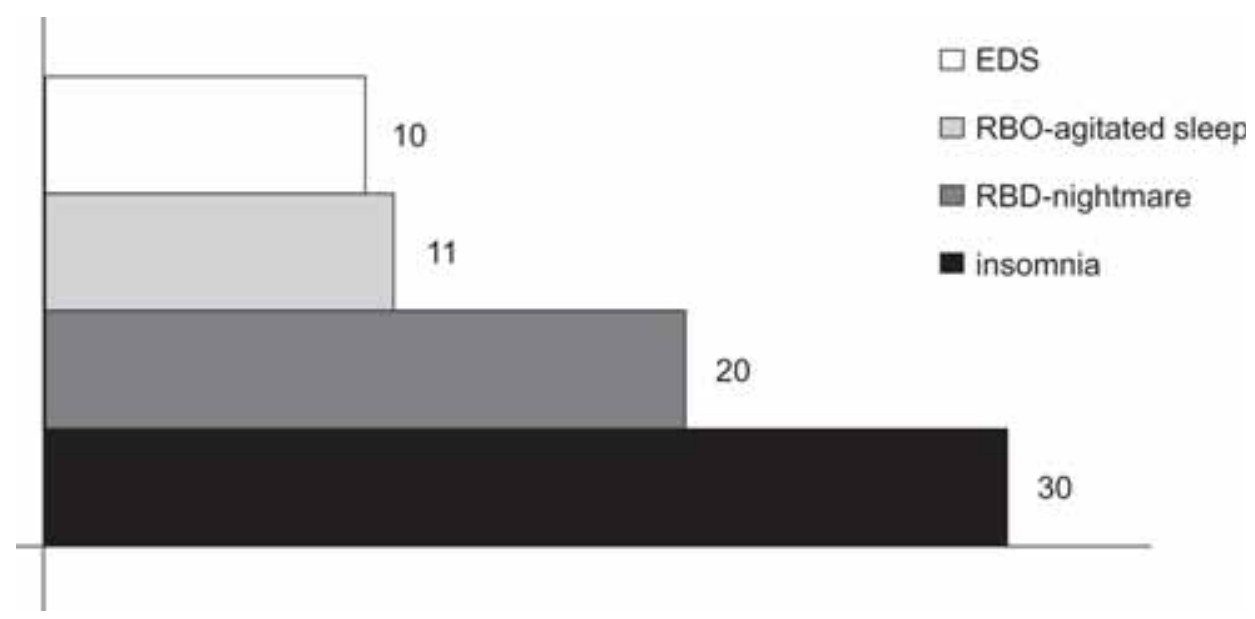

FIGURE 3. Frequency of sleep disturbances

$\mathrm{p}=0.5)$, exposure to toxic $(\mathrm{t}=1.11 ; \mathrm{p}=0.27)$, treatment $(t=-.20 ; p=0.84)$, the patient's age $(r=0.16)$, duration of illness $(r=0.18)$, the stage of the disease $(\mathrm{F}=2.19 ; \mathrm{p}=0.12)$, or type of the disease
$(\mathrm{F}=.90 ; \mathrm{p}=0.42)$. It has been established a statistical significant correlation between the presence of a positive family history and a high prevalence of psychiatric complaints $(\mathrm{t}=2.67 ; \mathrm{p}=0.03)$.

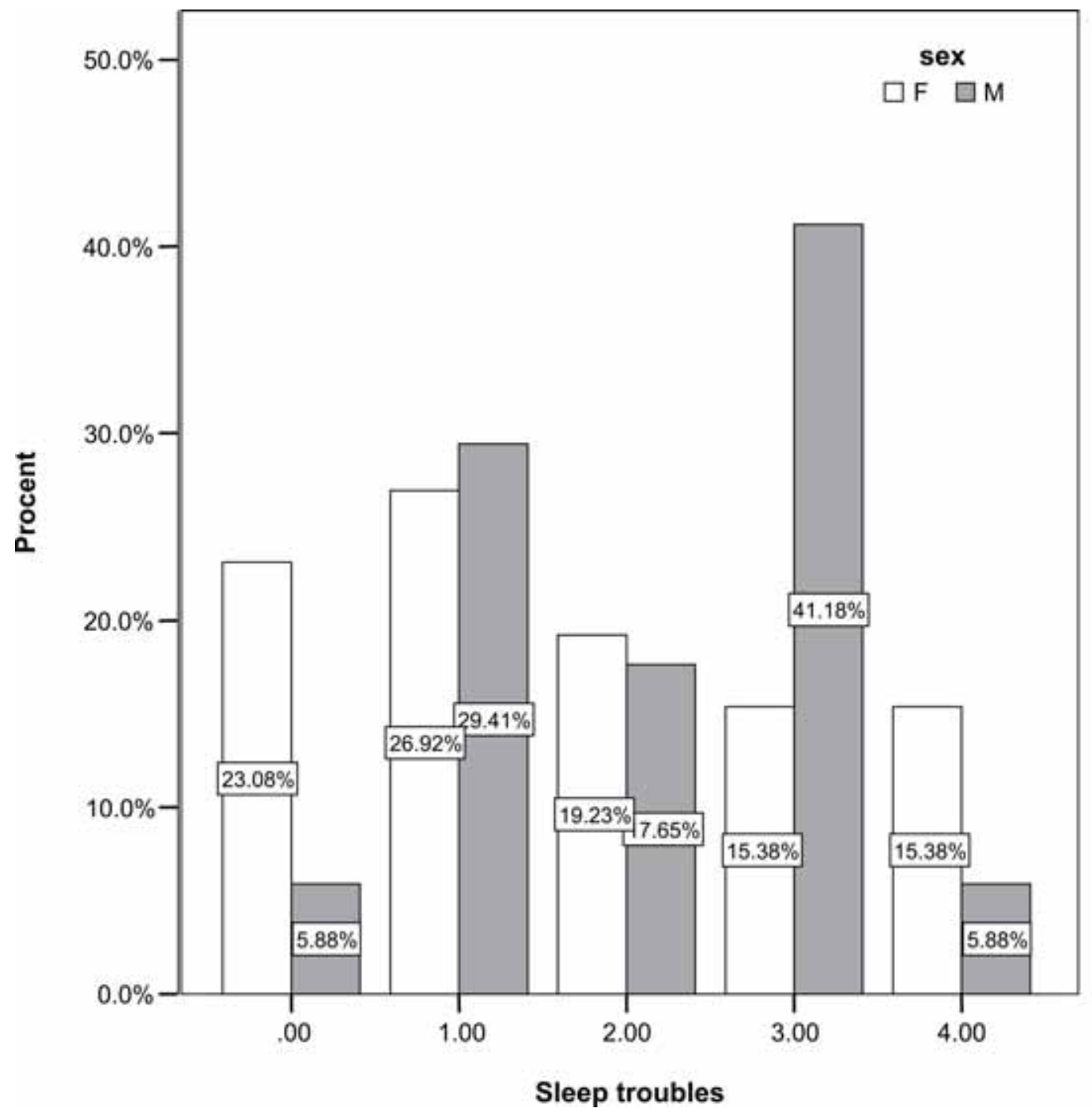

FIGURE 4. Distribution of sleep disorders according to gender 
The field of sleep disorders was well represented by insomnia, present in $69.76 \%$ of cases, which was followed by EDS and sleep with nightmares in equal percentages of $46.51 \%$. About a quarter of patients describe symptoms from category of RBD $-25.58 \%$.

Also, given complaints of impaired sleep did not differ significantly by gender $(t=.96 ; p=0.34)$, origin $(\mathrm{t}=-.10 ; \mathrm{p}=0.92)$, smoking $(\mathrm{t}=.88 ; \mathrm{p}=0.38)$, exposure to noxious $(\mathrm{t}=.66 ; \mathrm{p}=052)$, family history of $\mathrm{BP}(\mathrm{t}=-.15 ; \mathrm{p}=0.88)$, treatment $(\mathrm{t}=.69$; $\mathrm{p}=0.49)$, patient age $(\mathrm{r}=-0.04)$, disease duration $(\mathrm{r}=0.21)$.

Sleep disorders were detected in higher percentages in akinetic-rigid form of the disease $(\mathrm{F}=1.51$; $\mathrm{p}=.23$ ) and are related directly proportional to $\mathrm{H} \&$ $\mathrm{Y}$ stage of disease $(\mathrm{F}=6.64 ; \mathrm{p}=0,01)$. Disorders of sleep are often confounding: $41.15 \%$ of men presents combination of 3 types of symptoms, $17.65 \%$ have two symptoms and only one patient presents all 4 related symptoms. Only $5.88 \%$ of patients have no symptoms related to sleep disturbance. In women, a percentage of $26.92 \%$ presents one sleep disorder, $23.08 \%$ do not have impaired sleep and rest presents similar percentage by 2, 3 or 4 symptoms.

Psychiatric symptoms and sleep disorders do not correlate with disease duration, which suggests that may be present early in the course of the disease.

\section{DISCUSSIONS (17)}

Within the psychiatric field, the most prevalent symptom belonged to affective disorders-depression $-81,39 \%$, followed by disorders of attention and concentration - 79,06\%, memory impairment $74,41 \%$ and anxiety $-60,46 \%$.

Psychiatric complaints play an important role as there were no patients who did not present at least one psychiatric symptom and in most cases patients have described a complex of associated symptoms: roughly one third of the patients of both genders complain about 5 symptoms of each of this type, simultaneneously.

\section{REFERENCES}

1. Tolosa E., Poewe W. Premotor Parkinson disease. Neurology 2009; 72(Suppl):S1

2. Stern M.B., Siderowf A. Parkinson's at risk syndrome: can Parkinson's disease be predicted? Mov Disord 2010; 25 Suppl 1:S89-93.
The only statistically significant correlation was with family history of PD, which was associated with a higher prevalence of psychiatric symptoms.

Symptoms related to impaired sleep represent a special category of nonmotors complaints, that are increasingly in attention of clinicians, due to the importance they may have in the early diagnosis of disease, impact on their quality of life and impact of existing therapeutic possibilities. Disorders of sleep were detected in higher percentages in the akinetic-rigid form and are related directly with $\mathrm{H}$ \& Y stage of the disease. No other significant correlation was detected between the prevalence of sleep disorders and the other variables studied.

In literature, neuropsychiatric disturbances most commonly seen in the early stages are depression and anxiety; dementia and psychotic symptoms occur in advanced stage (8), which corresponds with the results of our study. Santamaria et al have determined presence of depression in $32 \%$ of patients with recent-onset PD and at $44 \%$ of patients, depression backdated few years motor manifestations (8). Anxiety can be present up to $62.9 \%$ of PD patients in the early stage and depression commonly coexist (8). In the study Priamo the psychiatric symptoms were most commonly reported (66.8\%) and was significantly more prevalent in women (16).

\section{CONCLUSION}

The most common psychiatric symptoms reported by the questionnaire even from the early stages were depression, attention and memory problems and those related to impaired sleep.

Sleep disturbances and psychiatric symptoms were not correlated with disease duration, which suggests that may be present early in the course of the disease.

The results presented highlights the importance of questioning the patients about nonmotor complaints from the very beginning of the diagnostic process.

3. Marek K., Jennings D., Tamagnan G. et al. Biomarkers for Parkinson's [corrected] disease: tools to assess Parkinson's disease onset and progression. Ann Neurol 2008; 64 Suppl 2:S111-21 
4. Morgan J.C., Mehta S.H., Sethi K.D. Biomarkers in Parkinson's disease. Curr Neurol Neurosci Rep 2010; 10(6):423-30

5. Tolosa E., Gaig C., Santamaría J. et al. Diagnosis and the premotor phase of Parkinson disease. Neurology 2009; 72 (7 Suppl): S12 - 20

6. de Lau L.M., Koudstaal P.J., Hofman A. et al. Subjective complaints precede Parkinson disease: the Rotterdam study. Arch Neurol 2006; 63(3):362-5.

7. Chaudhuri K.R., Martinez-Martin P., Schapira A.H. et al. International multicenter pilot study of the first comprehensive self completednonmotor symptoms questionnaire for Parkinson's disease: The NMSQuest study. Mov Disord 2006; 21(7):916-23.

8. Tolosa E., Gaig C., Santamaria J., Comta Y. Non-motor symptoms in the early motor stages of Parkinson's disease. In: Chaudhuri KR, Tolosa E, Schapira AH, Poewe W, editors. Non-motor symptoms of Parkinson's disease.1'st ed.New York: Oxford University Press; 2009

9. Leentjens A.F., Van den Akker M., Metsemakers J.F. et al. Higher incidence of depression preceding the onset of Parkinson's disease: a register study. Mov Disord 2003; 18(4):414-8

10. Boeve B.F., Silber M.H., Ferman T.J. et al. Association of REM sleep behavior disorder and neurodegenerative disease may reflect an underlying synucleinopathy. Mov Disord 2001;16(4):622-30
11. Schenck C.H., Mahowald M.W. REM sleep behavior disorder: clinical, developmental, and neuroscience perspectives 16 years after its formal identification in SLEEP. Sleep 2002; 25(2):120-38

12. Gagnon J.F., Bédard M.A., Fantini M.L. et al. REM sleep behavior disorder and REM sleep without atonia in Parkinson's disease. Neurology 2002; 59(4):585-9

13. Iranzo A., Santamaría J., Rye D.B. et al. Characteristics of idiopathic REM sleep behavior disorder and that associated with MSA and PD. Neurology 2005; 65(2):247-52

14. Vendette M., Gagnon J.F., Décary A. et al. REM sleep behavior disorder predicts cognitive impairment in Parkinson disease without dementia. Neurology 2007; 69(19):1843-9

15. Abbott R.D., Ross G.W., White L.R. et al. Excessive daytime sleepiness and subsequent development of Parkinson disease. Neurology 2005; 65(9):1442-6

16. Barone P., Antonini A., Colosimo C. et al. The PRIAMO study: $A$ multicenter assessment of nonmotor symptoms and their impact on quality of life in Parkinson's disease. Mov Disord. 2009; 24(11):1641-9.

17. PhD Thesis: Tohanean N. The role of biomarkers in the early diagnosis of Parkinson's disease. 2013 (doctoral dissertation)

Conflict of interest: none declared Financial support: none declared 